This is the final peer-reviewed accepted manuscript of:

M. Massaro, R. Amorati, G. Cavallaro, S. Guernelli, G. Lazzara, S. Milioto, R. Noto, P. Poma, S. Riela

Direct chemical grafted curcumin on halloysite nanotubes as dual-responsive prodrug for pharmacological applications

COLLOIDS AND SURFACES. B, BIOINTERFACES, 2016, vol. 140, p. 505-513

The final published version is available online at:

http://dx.doi.org/10.1016/j.colsurfb.2016.01.025

Rights / License:

The terms and conditions for the reuse of this version of the manuscript are specified in the publishing policy. For all terms of use and more information see the publisher's website.

This item was downloaded from IRIS Università di Bologna (https://cris.unibo.it/)

When citing, please refer to the published version. 


\title{
Direct chemical grafted curcumin on halloysite nanotubes as dual-responsive prodrug for pharmacological applications
}

\author{
M. Massaro, ${ }^{a}$ R. Amorati, ${ }^{b}$ G. Cavallaro, ${ }^{c}$ S. Guernelli, ${ }^{b}$ G. Lazzara,,${ }^{c}{ }^{*}$ S. Milioto,,${ }^{c}$ R. Noto,,${ }^{a}$ P. \\ Poma $^{\mathrm{d}}$ and S. Riela. ${ }^{\mathrm{a},{ }^{*}}$ \\ ${ }^{a}$ Dipartimento STEBICEF, Sez. Chimica, Università degli Studi di Palermo, Viale delle \\ Scienze, Parco d'Orleans II, Ed. 17, 90128 Palermo, Italy.E-mail: serena.riela@ unipa.it; Tel: +39-09123897546. \\ ${ }^{b}$ Dipartimento di Chimica "Giacomo Ciamician", Via S.Giacomo 11, Bologna, Italy. \\ bDipartimento di Fisica e Chimica, Universit' a degli Studi di Palermo, Viale delle \\ Scienze, Parco d'Orleans II, Ed. 17, 90128 Palermo, Italy.E-mail: giuseppe.lazzara@unipa.it; Tel:+39-09123897962. \\ Dipartimento di Scienze per la Promozione della Salute e Materno Infantile "G. D'Alessandro", Area Funzionale di \\ Farmacologia, Università di Palermo, Via \\ del Vespro 129, 90127 Palermo, Italy
}

Dedicated to memory of Prof. M. L. Turco Liveri

\section{ABSTRACT}

Given the complexity of drug delivery a combination of two or more stimuli in a single nanocarrier may provide more opportunities to maximize the efficiency of drug delivery. Along this line, we designed a prodrug based on halloysite nanotubes (HNT) that could respond to both intracellular glutathione $(\mathrm{GSH})$ and $\mathrm{pH}$ to release curcumin. In order to obtain the HNT-Cur produgs, HNTs were firstly functionalized with cysteamine through disulphide linkage and then curcumin molecules were chemically conjugated to the amino end groups of halloysite via Schiff's base formation. The succefull functionalization was proved by thermogravimetric analyis, FT-IR spectroscopy, dynamic light scattering measurements and scanning electron microscopy investigations. All these tecniques confirmed the presence of curcumin on HNT external surface. Moreover HNT-Cur prodrug showed dual stimuli-responsive controlled release upon exposure to GSH-rich or acidic environment. In order to verify the pharmacological properties of the new system., in vitro evaluation of its antiproliferative and antioxidant properties was performed.

KEYWORDS: halloysite nanotubes, curcumin, prodrug, antiproliferative activity, antioxidant properties.

\section{Introduction}

Halloysite nanotubes (HNTs), with a tubular structure like multi-walled carbon-nanotubes are an aluminosilicate with the ideal chemical formula of $\mathrm{Al}_{2} \mathrm{Si}_{2} \mathrm{O}_{5}(\mathrm{OH}) \cdot 2 \mathrm{H}_{2} \mathrm{O}$. The HNTs are natural clay with interesting physico-chemical properties [1].

In recent years HNTs due to abundant resources and low cost, have attracted considerable attention for applications in many fields [2]. 
Moreover HNTs have shown biocompatibility, no toxicity and no cytotoxic effects [3, 4]. Biocompatibility is the main prerequisite for safe usage of HNTs in medical products [5].

The external surface of HNTs is composed of Si-O-Si groups and the internal surface of a gibbsitelike array of $\mathrm{Al}-\mathrm{OH}$ groups. The peculiar surface chemistry allows the selective functionalization at the inner or outer side and the formation of a composite with hierarchical nanostructure [6, 7]. Incorporation of 5-aminosalicylic acid [8], diphenhydramine hydrochloride [9], diltiazem $\mathrm{HCl}$, propranolol $\mathrm{HCl}$ [10], tetracycline hydrochloride [11], dexamethasone, furosemide and nifedipine [12] and cardanol [13] into HNT was investigated and encouraging results were obtained. Recently it was reported new carrier systems for curcumin based on HNTs. In particular pH-[3] and thermoresponsive[14] carrier systems were developed.

Curcumin, a naturally active constituent extracted from the plants of the Curcuma longa, has a variety of biological activities and pharmacological actions $[15,16]$. The effects and reactions of curcumin have been the subject of investigation in the fields of biology, medicine, pharmacology, and in the food industry yielding a large number of publications for many years. The medical activity of curcumin has been known since ancient times. The beneficial effects can be attributed to its antioxidant activity which involve radical and peroxide scavenging, as well as metal chelating effect[17-22]; in addition curcumin shows anti-proliferative [23], antiapoptotic [24] and antiangiogenetic [25] properties.

Successful application of this compound is hampered, however, by the occurrence of some disadvantageous properties. Being hydrophobic in nature, curcumin is sparingly soluble in water. Moreover, it degrades rapidly under neutral or alkaline conditions, with a half-life shorter than 10 min in phosphate buffer solution at $\mathrm{pH} 7.2$ [26]. As a result, its bioavailability is poor, particularly after oral or topical administration [27]. Therefore, a carefully designed carrier could significantly facilitate curcumin delivery and broaden the range of its possible pharmaceutical applications.

To overcome these problems, in the years a number of drug delivery systems (DDS) was developed. These approaches include loading curcumin into liposomes or nanoparticles, forming curcuminphospholipid complex and synthesizing structural analogues of curcumin [27, 28].

However DDS have some disadvantages such as poor circulation stability, toxic side effects and low therapeutic efficacy [29-34].

Similar to other stimuli-triggered nanocarriers, stimuli-responsive prodrug has shown significant advantages in reducing side effects and enhancing antitumor activity due to tumor-specific drug release. Among them, in recent years acid-responsiveness is the most frequently used approach [35]. Acid-triggered drug release can be realized by the application of $\mathrm{pH}$-sensitive biodegradable chemical linkers, mainly including acid-labile acetal linkage, hydrazine bonds and silyl-ether bonds. 
Another stimulus used to achieve triggered drug release from prodrug is the redox gradient existing between the mildly oxidizing extracellular spaces and the reducing intracellular cytoplasm (higher reduction level) [36]. A disulfide bond is the most common chemical linker utilized to bridge polymers and anticancer drugs for reduction-responsive drug release [37, 38].

Inspired by our previous studies about halloysite carrier system, herein we report for the first time the synthesis and characterization of dual-stimuli-responsive halloysite-curcumin prodrug. Curcumin was covalently linked to HNT precursor through GSH- or pH-responsive bonds. With the final goal to extending the use of curcumin in pharmacological formulations kinetics release and biological properties associated with the prodrug were studied, too. Accordingly, the HNT-Cur prodrug synthesized was subjected to in vitro evaluation of its antiproliferative and antioxidant properties.

\section{Results and Discussion}

We started from synthesis of stimuli-responsive halloysite prodrug based on curcumin, as an anticancer and antioxidant model drug. Specifically, curcumin molecules were covalently conjugated with halloysite scaffold via $\mathrm{pH}$-responsive imine bond. In order to obtain dual stimuliresponsive prodrug, a halloysite scaffold bearing a GSH-responsive disulphide bond was synthesized (Scheme 1).

Pristine halloysite was transformed, as described [39], into f-HNT- SH 1 and it was covalently linked to cysteamine hydrochloride 2 (Scheme 1).

First of all, we investigated the synthetic procedure reported in literature for disulphide bond formation [40]. In these conditions, we obtained a product, which was analysed by TGA, after filtration, in order to evaluate the percentage of the loading. These conditions yielded a loading percentage as large as $3.7 \%$ (Table 1, Entry 1).

Afterwards, we investigated the same reaction performed by microwave irradiation (MW) in methanol or in solvent free conditions, as reported in Table 1 . In both cases after only $1 \mathrm{~h}$, a comparable loading to traditional heating was observed (Entries 2-3). The obtained results highlighted the importance of microwave irradiation in improving this synthesis.

The use of microwaves and solvent-free conditions indeed improved the linkage of compound on the HNT external surface, and accomplished it much more rapidly than traditional synthesis does. Since we have worked in solvent-free conditions, the reaction may be considered "green".

The covalent linkage of curcumin on compound $\mathbf{3}$ was carried out according to the experimental conditions reported in Table 1. We investigated firstly a synthetic procedure reported in literature for Schiff base synthesis.[41] Following this approach, i.e. a reaction time of $24 \mathrm{~h}$, a temperature of $78{ }^{\circ} \mathrm{C}$ using ethanol as solvent we obtained a loading of $0.88 \%$. Therefore, in order to improve the 
loading of curcumin on HNT surface, we performed the reaction under MW and in solvent-free condition (Entry 5); also in this case no improvement in loading was observed. Subsequently the reaction was performed using several solvents, with different ability to interact with microwaves. In particular we studied the effect of water, ethanol, THF and a mixture water/ethanol (1:1).

The results are reported in Table 1 (Entries 6-9) and the best experimental conditions were obtained in the presence of ethanol for an irradiation time of $1 \mathrm{~h}$ (Entry 8). The different percent loading obtained with the different solvents could be explained as a consequence of the different ability of the solvents to interact with microwaves and their different ability to solubilize curcumin. Ethanol and THF, indeed, even if can solubilize curcumin, are different respect to microwaves because have a different ability to convert microwave energy into heat as expressed by their $\tan \delta$ values ( $\tan \delta$ of 0.941 and 0.047 respectively) [42].

TGA experiments (Figure 1) provided the curcumin loading onto the HNT-Cur hybrid as well as the thermal behaviour of the organic moieties attached onto the nanoclay surface. As Figure 1a shows, the thermogram of compound $\mathbf{4}$ presents an excess of mass loss with respect to compound $\mathbf{3}$ in agreement with the attachment of the organic moiety.

In order to highlight the thermal stability of attached Cur in the composite material, TG curve of compound 3 was subtracted to that for compound 4. Figure $1 \mathrm{~b}$ highlights that the obtained mass change compared to that calculated for Cur pure sample scales down for the loading in the composite (Table 2, Entry 5). From such a comparison one can conclude that Cur is present in compound $\mathbf{4}$ and its thermal stability is not significantly altered in the composite material.

Furthermore, TGA results provided the stoichometry of compound 4 (HNT-Cur prodrug). Based on the curcumin loading $(2.90 \%)$ we determined that the mole ratio between curcumin and the amino groups linked onto HNT surface (compound 3) is 1:6.

The UV-vis absorption spectrum of HNT-Cur sample (See Figure S.1) shows the successful loading of drug molecules so as to obtain prodrug. As shown in Figure S.1, the ethanol solution of the prodrug exhibited the characteristic absorption of curcumin at about $420 \mathrm{~nm}$.

Compared to pristine HNT,[39] HNT-Cur exhibits the vibration bands for C-H stretching of methylene groups around $2960 \mathrm{~cm}^{-1}$ and $2865 \mathrm{~cm}^{-1}$ (Figure S.2). In addition the spectrum of HNTCur shows a band around $1511 \mathrm{~cm}^{-1}$ and $1457 \mathrm{~cm}^{-1}$ which are attributed to $-\mathrm{C}=\mathrm{N}$ and $\mathrm{C}-\mathrm{N}$ stretching, respectively, of the Schiff base. These findings provide evidence for the presence of organic moieties in compound HNT-Cur.

Based on the unaltered frequency of the stretching bands of the $\mathrm{OH}$ of the inner-surface $\mathrm{Al}-\mathrm{OH}$ groups, we can conclude that grafting has taken place only on the external surface of HNT and on the results from TG experiments. 
The morphology and the size of the HNTs prodrug were characterized by electron scanning microscopy (SEM) and dynamic light scattering (DLS).

Measurements of the particle size using DLS revealed an apparent hydrodynamic radius of $570 \mathrm{~nm}$ for HNT-Cur prodrug. This value is ca. 2 times larger than that expected for a single diffusive HNT particle $(240 \mathrm{~nm})$ [43]. The outer surface modification with Cur is expected to enhance the hydrophobic lateral interactions between nanotubes and therefore their aggregation which is likely reflected in the slow diffusion dynamics evidenced by the hydrodynamic radius increase. On the other hand SEM images on the dried samples show that the tubular morphology is preserved in the HNT-Cur prodrug as well as the characteristic sizes of the imaged nanotubes (Figure S.3).

\section{Kinetic release}

Recent studies reveal that prodrugs with a stimuli-responsive character are particularly promising for drug delivery. The prodrug designed in the present study was expected to display a $\mathrm{pH}$ and GSH reduction dual-responsive fashion.

It has been demonstrated that the concentration of GSH in tumor cells $(2-8 \mathrm{mM})$ is much higher than that in blood plasma $(1-2 \mu \mathrm{M})$ and compared to that in normal cells [44]. The unique intracellular redox potential has provided an inbuilt different release mechanism for controlled drug delivery.

The release of curcumin from HNT-Cur prodrug was investigated at simulated gastric and intestinal $\mathrm{pH}$ ( $\mathrm{pH} 1$ and 7.4 respectively). The method of dynamic dialysis was chosen to study the release behavior of curcumin from prodrug.

First of all we studied the controlled release of curcumin from HNT in phosphate buffered saline (pH 7.4) at $37{ }^{\circ} \mathrm{C}$ with or without GSH (Figure 2), resembling the difference of cellular environment between tumor cells and normal cells. Figure 5a shows that the drug release in the buffer containing $10 \mathrm{mM}$ GSH was significantly faster than that in the same buffer without GSH. Specifically, approximately $25 \%$ of curcumin was released from HNT in the first 100 min, followed by a prolonged release, in the presence of $10 \mathrm{mM}$ of GSH. In contrast, only about $5.0 \%$ of curcumin was released in the absence of GSH maintaining sink conditions. This latter result revealed that imine linkages were relative stable under neutral $\mathrm{pH}$ environment. The accelerated release in the presence of GSH was due to the dissociation of curcumin from the carrier caused by the reduction-triggered cleavage of disulphide linkage between HNT and curcumin via thioldisulphide exchange reactions. The results meant that the prodrug was stable under physiological conditions and therefore it is an ideal vehicle for targeted intracellular delivery. 
We also studied the controlled release of curcumin from HNT-Cur prodrug at $37{ }^{\circ} \mathrm{C}$ in $\mathrm{HCl} 0.1 \mathrm{~N}$ $(\mathrm{pH}$ 1). Figure $2 \mathrm{~b}$ shows that the release of curcumin under acidic environment was significantly faster than that in neutral environment because of the $\mathrm{pH}$-sensitivity of the imine bond.

The lower amount of curcumin released from HNT-Cur prodrug observed in neutral environment than in acidic one, is probably due to the different solubility of curcumin as a function of $\mathrm{pH}$. Indeed curcumin molecules $\left(\mathrm{pK}_{\mathrm{a} 1}=8.38, \mathrm{pK}_{\mathrm{a} 2}=9.88\right.$ and $\left.\mathrm{pK}_{\mathrm{a} 3}=10.51\right)$ can exist as cationic, neutral or anionic species in acidic, neutral or alkaline solution, respectively [3]. Therefore, curcumin can be easily released at $\mathrm{pH} 1$ in its cationic form (Figure 2b), resulting in a higher release as compared with that at $\mathrm{pH} 7.4$ (Figure 2a), given that Cur is not soluble at nearly neutral $\mathrm{pH}$.

In order to study the release mechanism, the release data were analysed according to the Power fit equation:

$$
\frac{M_{t}}{M_{\infty}}=k t^{n}
$$

where $M_{t} / M_{\infty}$ was the fractional amount of the curcumin released at time $t, k$ was a characteristic constant, and $n$ was the release exponent, depending on the release mechanism and the geometry of the device.

The constants $n$ and $k$ were calculated according to the fitted curves of drug release profiles.

The results showed that in both cases $\mathrm{R}^{2}$ was higher than 0.98 and the value of kinetic constant in acidic environment was lower than $96 \%$ than that at $\mathrm{pH} 7.4$ (Table S.1). The latter result indicated that different release mechanisms occurred as function of $\mathrm{pH}$.

In particular, at $\mathrm{pH} 7.4$ we probably observed the fast diffusion mechanism of curcumin $(k=14 \pm 1$ $\min ^{-1}$ ) through the dialysis membrane as a consequence of the instantaneous disulphide bond reduction by GSH. In contrast, at $\mathrm{pH} 1$, HNT-Cur prodrug exhibited a sustained-release feature $(k=$ $0.6 \pm 0.2 \mathrm{~min}^{-1}$ ) and we observed that curcumin was released slowly from the matrix probably due to the hydrolysis of imine bond. To confirm these hypothesis in Figure $2 b$ is also reported the release of pure curcumin [45]. It is approximately $91 \%$ within $6 \mathrm{~h}$ indicating that the release of Cur from solution through the dialysis membrane is a fast process.

\section{Antiproliferative activity}

Hepatocellular carcinoma (HCC) represents the fifth most common cancer worldwide and the third leading cause of cancer-related mortality globally, maintaining a dismal prognosis since intermediate and advanced stages still account for a large percentage of cases [46-48]. The majority of patients with HCC dies within 1 year after the diagnosis of their disease. For these reasons we investigated the antiproliferative activity of HNT-Cur prodrug against HA22T/VGH and Hep3B 
cell lines by vitality count. The cells were incubated with HNT, Cur and HNT-Cur prodrug for $48 \mathrm{~h}$. The results revealed that HNTs were not-toxic to HA22T/VGH and Hep3B cells at a concentrations of up $100 \mathrm{mg} / \mathrm{mL}$, indicating that HNT possessed excellent biocompatibility as previously reported [3]. Free curcumin had no effect on cell viability probably due to its low solubility in physiological medium, which limits its clinical application [3].

Otherwise HNT-prodrug exhibited a significant cytotoxicity to tumor cells after incubation for $48 \mathrm{~h}$. Only $22 \%$ of cells remained survival with the concentration up to $50 \mu \mathrm{M}$ for Hep3B and $16 \%$ for HA22T/VGH respectively, and for Hep3B, no viability was observed at higher concentration (100 $\mu \mathrm{M}$ ) (Figure 3). The accelerated curcumin release from the nanoparticle triggered by intracellular GSH should be responsible for the increased cytotoxicity of the HNT-Cur.

\section{Antioxidant activity}

The antioxidant activity of curcumin comes by virtue of its chemical structure. The antioxidant activity results from two structural elements, namely, the phenolic $-\mathrm{OH}$ groups and the $\mathrm{CH}_{2}$ group of the $\beta$-diketone moiety, that in solution is in equilibrium with its enol tautomer [49-51]. Curcumin has been shown to inhibit lipid peroxidation and to manifest free radical-scavenging activity, in particular towards oxygen and nitrogen-centered free radicals. Recently it was reported that curcumin is able to inhibit the activity of inducible nitric oxide synthase (iNOS), an enzyme found in macrophages that generates large amounts of NO in response to an oxidative environment. The anti-oxidant and free-radical quenching properties are related to the inhibitory effects of the compound on the initial stages of carcinogenesis [52].

\section{Autoxidation experiments}

The antioxidant activity of HNT-Cur and curcumin was evaluated by measuring the rate constant $\left(k_{i n h}\right)$ for the reaction with peroxyl radicals $(\mathrm{ROO} \bullet)$, that are responsible for the propagation step of peroxidation processes in many natural and man-made materials [53]. The values of $k_{\text {inh }}$ (equation 5, where $\mathrm{AH}$ is an antioxidant) were determined by studying the inhibition of the thermally initiated autoxidation of cumene (RH) (Eqs. 2-7) under controlled conditions, using chlorobenzene or acetonitrile as a solvent, which are employed to simulate the lipophilic interior of biological membranes $[54,55]$.

$$
\begin{aligned}
& \text { Initiator } \stackrel{R_{i}}{\longrightarrow} R^{\bullet} \\
& R^{\bullet}+O_{2} \rightarrow R O O^{\bullet} \\
& R O O^{\bullet}+R H \stackrel{k_{p}}{\longrightarrow} R^{\bullet}+R O O H
\end{aligned}
$$


The reactions were performed at $303 \mathrm{~K}$ using azobis(isobutyronitrile) (AIBN) as initiator, and were followed by monitoring the oxygen consumption in an oxygen-uptake apparatus based on a differential pressure transducer. Typical plots of $\mathrm{O}_{2}$ consumption are reported in Figure 4. In the presence of antioxidants, oxidation of the substrate and oxygen consumption are much slower than in their absence, and a clear inhibition period is observed [56]. The values of $k_{\text {inh }}$ and the number of radicals trapped by each antioxidant molecule $(n)$ are reported in Table 2 .

\section{DPPH• bleaching experiments}

The radical trapping ability of HNT-Cur and curcumin was also assessed by studying the reaction with 2,2-di(4-tert-octylphenyl)-1-picrylhydrazyl radical (herein: oDPPH•), that is a lipophilic analogue of the persistent coloured 2,2-diphenyl-1-picrylhydrazyl radical. These radicals are commonly used to evaluate the antioxidant properties of natural products, as the purple free radical is transformed by reductants to the yellow hydrazine through a formal hydrogen atom transfer reaction (see Scheme 3) [57, 58]. For these measurements we decided to use the derivative having two aromatic rings substituted with alkyl groups in order to minimize its incorporation into the polar cavity of the nanotubes. After mixing an excess of oDPPH - with curcumin, or with HNT-Cur in $\mathrm{MeCN}$, the absorbance at $537 \mathrm{~nm}$ of oDPPH $\bullet$ decreased, while the addition of bare HNT did not modify the absorption spectrum (see Table 2). The absorption spectra were corrected by subtracting the scattering of the nanoparticles, and the contribution given by the oxidized curcumin that gave a broad absorption at about $510 \mathrm{~nm}$. The number of oDPPH • radicals quenched by each molecule of curcumin, free or linked to HNT, were measured from absorbance readings at the end of the reaction, as reported in Table 3.

Results reported in Tables 2-3 and Figure 7, show that HNT-Cur is endowed with antioxidant activity, while bare HNT is not able to trap radicals. The $k_{\text {inh }}$ values of HNT-Cur, shown in Table 3 , are very similar to those of curcumin. The values of $k_{i n h}$ of HNT-Cur and curcumin decrease when changing the solvent from $\mathrm{PhCl}$ to $\mathrm{MeCN}$, as effect of the formation of $\mathrm{H}$-bond between the reactive phenolic $\mathrm{OH}$ and the solvent, which hinders the $\mathrm{H}$-atom abstraction by peroxyl radicals [59]. These results indicate that HNT-Cur and curcumin trap peroxyl radicals through a $\mathrm{H}$-atom transfer from the phenolic $\mathrm{OH}$. 
The stoichiometry of radical trapping is an important parameter to describe the antioxidant activity of phenols. The number of radicals trapped by each molecule of curcumin is $\sim 3.7$ and 4 for ROO• and $\mathrm{oDPPH} \bullet$ respectively. These values are very near to $n=4$, that is the stoichiometry expected for a bifunctional phenolic antioxidant, having two isolated phenolic moieties, as each phenol can trap two radicals through reactions 5 and 6. In the case of HNT-Cur, the $n$ value is about 2 in both in the case of ROO• and oDPPH• radicals, suggesting a more complex mechanism. A stoichiometry decrease of the radical trapping was previously observed in the case of phenols linked to nanoparticles $[54,60]$ and was explained as effect of the self-decay of the radical of the antioxidant (A• in equation 5) which form A-A dimers instead of trapping a second radical [60]. Therefore, curcumin covalently linked to HNT maintains its reactivity toward radicals, whereas its stoichiometry is halved with respect to free curcumin.

\section{Experimental Section}

\section{Materials and instrumentation}

All needed reagents were used as purchased (Aldrich), without further purification. f-HNT-SH 1 was synthesized as previous reported [39].

Thermogravimetric analyses were performed on a Q5000 IR apparatus (TA Instruments) under a nitrogen flow of $25 \mathrm{~cm}^{3} \cdot \mathrm{min}^{-1}$ for the sample and $10 \mathrm{~cm}^{3} \cdot \mathrm{min}^{-1}$ for the balance. The weight of each sample was ca. $10 \mathrm{mg}$. Measurements were carried out by heating the sample from r.t. up to $900{ }^{\circ} \mathrm{C}$ at a rate of $10{ }^{\circ} \mathrm{C} \cdot \mathrm{min}^{-1}$.

An AESEM FEI QUANTA 200F microscope apparatus was used to study the morphology of the functionalized HNTs. Before each experiment, the sample was coated with gold under argon by means of an Edwards Sputter Coater S150A, in order to avoid charging under electron beam.

IR spectra $(\mathrm{KBr})$ were recorded using an Agilent Technologies Cary 630 FT-IR spectrometer. Specimens for measurements were prepared by mixing $5 \mathrm{mg}$ of the sample powder with $100 \mathrm{mg}$ of $\mathrm{KBr}$.

MW-assisted synthesis were carried out with a CEM DISCOVER mono-mode system in closed vessel.

DLS measurements were performed by means of a ZETASIZER NANO ZS90 (Malvern Instruments).

UV-vis spectra were performed with a Beckmann DU 650 spectrometer.

Spectroscopic measurements for determination of DPPH scavenging were performed on a Jasco V$550 \mathrm{UV}-\mathrm{V}$ is spectrophotometer with $1.0 \mathrm{~cm}$ quartz cells at a temperature of $298 \pm 0.1 \mathrm{~K}$ 
The dispersions were sonicated using an ultrasound bath VWR Ultrasonic Cleaner (power $200 \mathrm{~W}$ and frequency $75 \mathrm{MHz}$ ).

\section{Synthesis of compound 3}

Thiol functionalized HNTs $1(0.10 \mathrm{~g})$ were weighted in a MW test tube provided with a cap and cysteamine hydrochloride $(0.4 \mathrm{mmol})$ was added. To this mixture a catalytic amount of $\mathrm{I}_{2}$ was added. The mixture was inserted in the MW apparatus at $50{ }^{\circ} \mathrm{C}$ and a power of $1 \mathrm{~W}$, under constant stirring, for $1 \mathrm{~h}$. The solid was filtered off and rinsed with $\mathrm{MeOH}$. Then, the precipitate was dispersed in a $\mathrm{K}_{2} \mathrm{CO}_{3}$ solution, filtered off, rinsed with $\mathrm{H}_{2} \mathrm{O}$ and dried at $80{ }^{\circ} \mathrm{C}$ overnight.

\section{Synthesis of compound 4}

Compound $3(100 \mathrm{mg})$ and curcumin $(100 \mathrm{mg}, 0.27 \mathrm{mmol})$ were dissolved in EtOH (1 mL) in a MW test tube provided with a cap. The dispersion was inserted in the MW apparatus at $80{ }^{\circ} \mathrm{C}$ and a power of $20 \mathrm{~W}$, under constant stirring for $1 \mathrm{~h}$. The solid was filtered off, rinsed with $\mathrm{MeOH}$ and dried at $80^{\circ} \mathrm{C}$ overnight.

\section{Kinetic Release}

The release of curcumin from the HNT-Cur prodrug was performing as follows: $25 \mathrm{mg}$ of the sample were dispersed in $1 \mathrm{~mL}$ of dissolution medium and transferred into a dialysis membrane (Medicell International Ltd MWCO 12-14000 with diameter of 21.5 mm). The membrane was then put in a round bottom flask containing $10 \mathrm{~mL}$ of the release medium at $37^{\circ} \mathrm{C}$ and stirred.

Two different media $(0.1 \mathrm{M} \mathrm{HCl}$ and phosphate buffer $\mathrm{pH} 7.4$, respectively) were considered in order to evaluate the influence of $\mathrm{pH}$ on the release behaviour of the drug.

At fixed times, $1 \mathrm{~mL}$ of the release medium was withdrawn and analysed. To keep constant the volume of the release medium $1 \mathrm{~mL}$ of fresh solution $(0.1 \mathrm{M} \mathrm{HCl}, \mathrm{pH} 7.4$ buffer $)$ was added each time to replace the withdrawn one.

The curcumin concentrations in the solution were determined by UV-vis spectrophotometry using the Lambert-Beer law.

Total amounts of drug released $\left(F_{t}\right)$ were calculated as follows:

$$
F_{t}=V_{m} C_{t}+\sum_{i=0}^{t-1} V_{a} C_{i}
$$

where $V_{m}$ and $C_{t}$ are volume and concentration of the drug at time $t . V_{a}$ is the volume of the sample withdrawn and $C_{i}$ is drug concentration at time $i(i<t)$.

\section{Cell culture}

Hep3B and HA22T/VGH are a poorly differentiated hepatoma cell lines which contain HBV integrants. It was cultured in Roswell Park Memorial Institute (RPMI) 1640 (HyClone Europe Ltd., Cramlington, UK) supplemented with $10 \%$ heat-inactivated fetal calf serum, 2 mM L-glutamine, 1 
$\mathrm{mM}$ sodium pyruvate, 100 units $/ \mathrm{mL}$ penicillin and $100 \mathrm{mg} / \mathrm{mL}$ streptomycin (all reagents were from HyClone Europe) in a humidified atmosphere at $37{ }^{\circ} \mathrm{C}$ in $5 \% \mathrm{CO}_{2}$. Cells having a narrow range of passage number were used for all experiments.

\section{Vitality count}

The cells were seeded at $1 \times 10^{5}$ cells/well onto 24 -well plates and then incubated overnight. At time 0, medium was replaced with fresh complete medium and curcumin, HNT and HNT-Cur prodrug thereof were added in concentrations as indicated. After $48 \mathrm{~h}$ of treatment, the cells were trypsinized and counted by Burker chamber. Vitality was expressed as a percentage respect of the cells not treated.

\section{Kinetics with peroxyl radicals}

The chain-breaking antioxidant activity of the title compounds was evaluated by studying the inhibition of the thermally initiated autoxidation of cumene in chlorobenzene. Autoxidation experiments were performed by measuring the $\mathrm{O}_{2}$ consumption by a gas-uptake recording apparatus built in our laboratory based on a Validyne DP15 differential pressure transducer. In a typical experiment, an air-saturated mixture of cumene $(5.3 \mathrm{M})$ in chlorobenzene or $\mathrm{MeCN}$ containing AIBN $\left(2.5 \times 10^{-2} \mathrm{M}\right)$ was equilibrated with the reference solution containing also an excess of 2,2,5,7,8-pentamethyl-6-chromanol $(\alpha-\mathrm{TOH})$ in the same solvent at $30^{\circ} \mathrm{C}$. After equilibration, 50 $150 \mu \mathrm{L}$ of a concentrated solution of the antioxidant was injected into the sample flask, and the oxygen consumption in the sample was measured. From the slope of the oxygen consumption during the inhibited period (see Figure 5), $k_{\text {inh }}$ values were obtained by using equation 8 while the $n$ coefficient was determined from the length of the inhibited period $\left(T_{i n h}\right)$ by using equation 9 .

$$
\begin{aligned}
& \Delta\left[O_{2}\right] t=-\frac{k_{p}}{k_{i n h}} \cdot[\text { cumene }] \cdot \ln \left(\frac{1-t}{T_{i n h}}\right) \\
& n=\frac{R_{i} \cdot T_{i n h}}{[\text { antioxidant }]} \\
& R_{i}=\frac{2[a-T O H]}{T_{i n h}}
\end{aligned}
$$

The $k_{p}$ values of cumene at $303 \mathrm{~K}$ is $41 \mathrm{M}^{-1} \mathrm{~s}^{-1}$. The value of $R_{i}$ was determined by using $\alpha-\mathrm{TOH}$ $(n=2)$ as a reference [55].

\section{Determination of DPPH scavenging}

Two series of measurements were performed using as antioxidant free curcumin (Cur) and curcumin linked to the outer surface of halloysite nanotubes (HNT-Cur). The reaction mixtures were prepared by adding to the oDPPH• radical (2,2-di(4-tert-octylphenyl)-1-picryl-hydrazyl free 
radical) in acetonitrile $\left(1.0 \times 10^{-4}\right.$ and $\left.5.0 \times 10^{-5} \mathrm{M}\right)$ different concentrations of curcumin (from 1.52 $\mathrm{x} 10^{-5}$ to $7.60 \times 10^{-6} \mathrm{M}$ ). Total volume of the reaction mixture was $2 \mathrm{ml}$. For each system, free curcumin, used a reference, and linked curcumin, concentrations were expressed as ratio (number of moles of DPPH/number of moles of antioxidant) Absorption of oDPPH• was determined by recording spectra in sequence until the absorbance was unchanged and against a blank solution that contained only acetonitrile $\left(\lambda_{\max }=537 \mathrm{~nm}, \varepsilon=9200 \pm 50\right)$.

\section{Conclusions}

We have developed a dual stimuli-responsive halloysite prodrug by covalently linking curcumin to HNT precursor through GSH- or $\mathrm{pH}$-responsive bonds, respectively.

The successful functionalization of halloysite outer surface with curcumin was evidenced by thermogravimetry. Thermal behaviour of curcumin is not altered in the hybrid nanomaterial, which preserves the peculiar tubular morphology of halloysite. Aqueous diffusion dynamics of halloysite nanotubes is slowed by the outer surface modification in agreement with the hydrophobic interactions between the attached organic moieties.

Halloysite prodrug showed relatively good stability under normal physiological conditions. Dramatically enhanced release of curcumin was observed upon exposure of this prodrug to GSHrich or acidic conditions similar to the cellular microenvironment of hepatocancer cells. The antiproliferative in vitro studies on two cell lines of hepatocellular carcinoma showed that HNT-Cur prodrug induced high cytotoxicity respect to the free curcumin or pristine HNT. The possibility of increase curcumin release from the nanoparticle by intracellular GSH appears very interesting, especially for HCC, because GSH is typically concentrated in hepatic cells.

Furthermore HNT-Cur prodrug retains its antioxidant characteristics, that could be crucial in anticancer therapy and open the possibility for further investigations and developments.

\section{Acknowledgements}

The work was financially supported by the University of Palermo, PRIN 2010-2011 (prot. 2010329WPF), FIRB 2012 (prot. RBFR12ETL5), PON-TECLA (PON03PE_00214_1) and from the University of Bologna (FARB project FFBO123154).

\section{References}

[1] Y. Lvov, W. Wang, L. Zhang, R. Fakhrullin, Adv. Mater., (2015) n/a-n/a.

[2] Q. Wang, J. Zhang, Y. Zheng, A. Wang, Colloids Surf. B. Biointerfaces, 113 (2014) 51-58.

[3] S. Riela, M. Massaro, C.G. Colletti, A. Bommarito, C. Giordano, S. Milioto, R. Noto, P. Poma, G. Lazzara, Int. J. Pharm., 475 (2014) 613-623. 
[4] V. Vergaro, E. Abdullayev, Y.M. Lvov, A. Zeitoun, R. Cingolani, R. Rinaldi, S. Leporatti, Biomacromolecules, 11 (2010) 820-826.

[5] Y. Lvov, A. Aerov, R. Fakhrullin, Adv. Colloid Interface Sci., 207 (2014) 189-198.

[6] W.O. Yah, A. Takahara, Y.M. Lvov, J. Am. Chem. Soc., 134 (2012) 1853-1859.

[7] G. Cavallaro, G. Lazzara, S. Milioto, F. Parisi, V. Sanzillo, ACS Appl. Mater. Interf., 6 (2014) 606-612.

[8] M.-T. Viseras, C. Aguzzi, P. Cerezo, G. Cultrone, C. Viseras, J. Microencaps., 26 (2009) 279286.

[9] A. Ghebaur, S.A. Garea, H. Iovu, Int. J. Pharm., 436 (2012) 568-573.

[10] S.R. Levis, P.B. Deasy, Int. J. Pharm., 253 (2003) 145-157.

[11] B.P.G.Y.L.R. R. Price, J. Microencaps., 18 (2001) 713-722.

[12] N.G. Veerabadran, R.R. Price, Y.M. Lvov, Nano, 2 (2007) 115-120.

[13] M. Massaro, C.G. Colletti, R. Noto, S. Riela, P. Poma, S. Guernelli, F. Parisi, S. Milioto, G. Lazzara, Int. J. Pharm., 478 (2015) 476-485.

[14] G. Cavallaro, G. Lazzara, M. Massaro, S. Milioto, R. Noto, F. Parisi, S. Riela, J. Phys. Chem. C, 119 (2015) 8944-8951.

[15] C. Araújo, L. Leon, Mem. Inst. Oswaldo Cruz, 96 (2001) 723-728.

[16] C.-H. Hsu, A.-L. Cheng, CLINICAL STUDIES WITH CURCUMIN, in: B. Aggarwal, Y.-J. Surh, S. Shishodia (Eds.) The Molecular Targets and Therapeutic Uses of Curcumin in Health and Disease, Springer US2007, pp. 471-480.

[17] M.K. Unnikrishnan, M.N.A. Rao, Mol. Cell. Biochem., 146 (1995) 35-37.

[18] R.J. Anto, G. Kuttan, K.V.D. Babu, K.N. Rajasekharan, R. Kuttan, Int. J. Pharm., 131 (1996) $1-7$.

[19] T. Ak, İ. Gülçin, Chem. Biol. Interact., 174 (2008) 27-37.

[20] N. Agnihotri, P.C. Mishra, J. Phys. Chem. A, 115 (2011) 14221-14232.

[21] M. Borsari, E. Ferrari, R. Grandi, M. Saladini, Inorg. Chim. Acta, 328 (2002) 61-68.

[22] V. Kant, A. Gopal, N.N. Pathak, P. Kumar, S.K. Tandan, D. Kumar, Int. Immunopharmacol., 20 (2014) 322-330.

[23] H. Choi, Y.-S. Chun, S.-W. Kim, M.-S. Kim, J.-W. Park, Mol. Pharmacol., 70 (2006) 16641671.

[24] S. Aggarwal, H. Ichikawa, Y. Takada, S.K. Sandur, S. Shishodia, B.B. Aggarwal, Mol. Pharmacol., 69 (2006) 195-206.

[25] Y.G. Lin, A.B. Kunnumakkara, A. Nair, W.M. Merritt, L.Y. Han, G.N. Armaiz-Pena, A.A. Kamat, W.A. Spannuth, D.M. Gershenson, S.K. Lutgendorf, B.B. Aggarwal, A.K. Sood, Clin. Cancer. Res., 13 (2007) 3423-3430.

[26] B.T. Kurien, A. Singh, H. Matsumoto, R.H. Scofield, Assay Drug Dev. Technol., 5 (2007) 567-576.

[27] P. Anand, A.B. Kunnumakkara, R.A. Newman, B.B. Aggarwal, Mol. Pharm., 4 (2007) 807818.

[28] T. Marczylo, R. Verschoyle, D. Cooke, P. Morazzoni, W. Steward, A. Gescher, Cancer Chemother. Pharmacol., 60 (2007) 171-177.

[29] S. Yu, J. Ding, C. He, Y. Cao, W. Xu, X. Chen, Adv. Healthcare Mater., 3 (2014) 752-760.

[30] E.K.-H. Chow, D. Ho, Cancer Nanomedicine: From Drug Delivery to Imaging, 2013.

[31] Y. Wang, M.S. Shim, N.S. Levinson, H.-W. Sung, Y. Xia, Adv. Funct. Mater., 24 (2014) 4206-4220. 
[32] S. Bonacchi, D. Genovese, R. Juris, M. Montalti, L. Prodi, E. Rampazzo, N. Zaccheroni, Angew. Chem. Int. Ed., 50 (2011) 4056-4066.

[33] M. Montalti, L. Prodi, E. Rampazzo, N. Zaccheroni, Chem. Soc. Rev., 43 (2014) 4243-4268.

[34] J. Nicolas, S. Mura, D. Brambilla, N. Mackiewicz, P. Couvreur, Chem. Soc. Rev., 42 (2013) 1147-1235.

[35] S.R. MacEwan, D.J. Callahan, A. Chilkoti, Nanomedicine, 5 (2010) 793-806.

[36] Q. Yin, J. Shen, Z. Zhang, H. Yu, Y. Li, Adv. Drug Del. Rev., 65 (2013) 1699-1715.

[37] Z. Zhou, X. Ma, E. Jin, J. Tang, M. Sui, Y. Shen, E.A. Van Kirk, W.J. Murdoch, M. Radosz, Biomaterials, 34 (2013) 5722-5735.

[38] A.J. van der Vlies, U. Hasegawa, J.A. Hubbell, Mol. Pharm., 9 (2012) 2812-2818.

[39] M. Massaro, S. Riela, G. Cavallaro, M. Gruttadauria, S. Milioto, R. Noto, G. Lazzara, J. Organomet. Chem., 749 (2014) 410-415.

[40] M.W. Ambrogio, T.A. Pecorelli, K. Patel, N.M. Khashab, A. Trabolsi, H.A. Khatib, Y.Y. Botros, J.I. Zink, J.F. Stoddart, Org. Lett., 12 (2010) 3304-3307.

[41] T. Chandrasekar, N. Pravin, N. Raman, Inorg. Chem. Commun., 43 (2014) 45-50.

[42] The ability of a specific solvent to convert microwave energy into heat at a given frequency and temperature is determined by the so-called loss tangent $(\tan \delta)$, expressed as the quotient, $\tan$ $\delta=\mathrm{e}^{\prime} / \mathrm{e}$, where $\mathrm{e} "$ is the dielectric loss, indicative of the efficiency with which electromagnetic radiation is converted into heat, and $\mathrm{e}^{\prime}$ is the dielectric constant, describing the ability of molecules to be polarized by the electric field. In general, solvents used for microwave synthesis can be classified as high $(\tan \delta>0.5)$, medium $(\tan \delta 0.1-0.5)$ and low microwave absorbing $(\tan \delta<0.1)$.

[43] G. Cavallaro, G. Lazzara, S. Milioto, Langmuir, 27 (2011) 1158-1167.

[44] Z. Xu, D. Wang, S. Xu, X. Liu, X. Zhang, H. Zhang, Chem. Asian J., 9 (2014) 199-205.

[45] M. Sun, Y. Gao, C. Guo, F. Cao, Z. Song, Y. Xi, A. Yu, A. Li, G. Zhai, J. Nanopart. Res., 12 (2010) 3111-3122.

[46] M. Bondi, A. Azzolina, E. Craparo, C. Botto, E. Amore, G. Giammona, M. Cervello, Journal of Nanobiotechnology, 12 (2014) 21.

[47] E.F. Craparo, C. Sardo, R. Serio, M.G. Zizzo, M.L. Bondì, G. Giammona, G. Cavallaro, Int. J. Pharm., 466 (2014) 172-180.

[48] A. Forner, J.M. Llovet, J. Bruix, The Lancet, 379 (2012) 1245-1255.

[49] P. Malik;, T.K. Mukherjee, Hindawi Publishing Corporation Chinese Journal of Biology, 2014 (2014).

[50] K.I. Priyadarsini, D.K. Maity, G.H. Naik, M.S. Kumar, M.K. Unnikrishnan, J.G. Satav, H. Mohan, Free Radical Biol. Med., 35 (2003) 475-484.

[51] K.S. Parvathy, P.S. Negi, P. Srinivas, Food Chem., 115 (2009) 265-271.

[52] R. Wilken, M. Veena, M. Wang, E. Srivatsan, Mol. Cancer, 10 (2011) 12.

[53] R. Amorati, L. Valgimigli, Free Radical Res., 49 (2015) 633-649.

[54] C. Viglianisi, V. Di Pilla, S. Menichetti, V.M. Rotello, G. Candiani, C. Malloggi, R. Amorati, Chem. Eur. J., 20 (2014) 6857-6860.

[55] G.W. Burton, K.U. Ingold, J. Am. Chem. Soc., 103 (1981) 6472-6477.

[56] H. Johansson, D. Shanks, L. Engman, R. Amorati, G.F. Pedulli, L. Valgimigli, J. Organomet. Chem., 75 (2010) 7535-7541.

[57] K. Mishra, H. Ojha, N.K. Chaudhury, Food Chem., 130 (2012) 1036-1043.

[58] R. Amorati, S. Menichetti, C. Viglianisi, M.C. Foti, Chem. Commun., 48 (2012) 11904-11906. 
[59] R. Amorati, L. Valgimigli, Org. Biomol. Chem., 10 (2012) 4147-4158.

[60] Y. Deligiannakis, G.A. Sotiriou, S.E. Pratsinis, ACS Appl. Mater. Interf., 4 (2012) 6609-6617.

Figures and Tables

1)

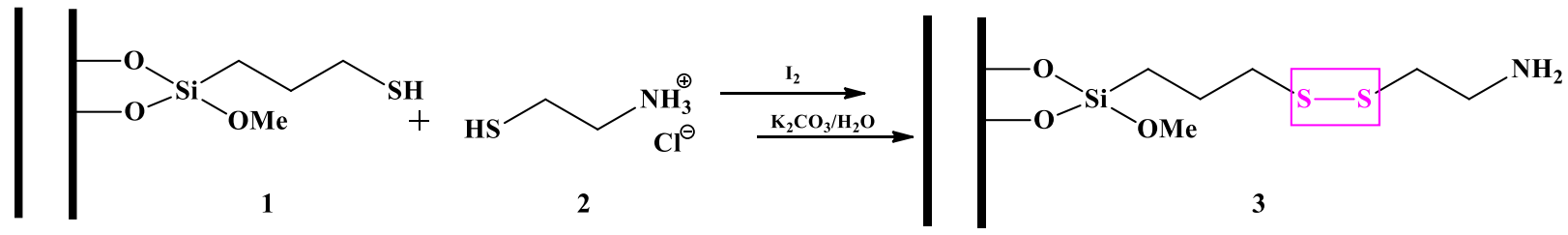

2)

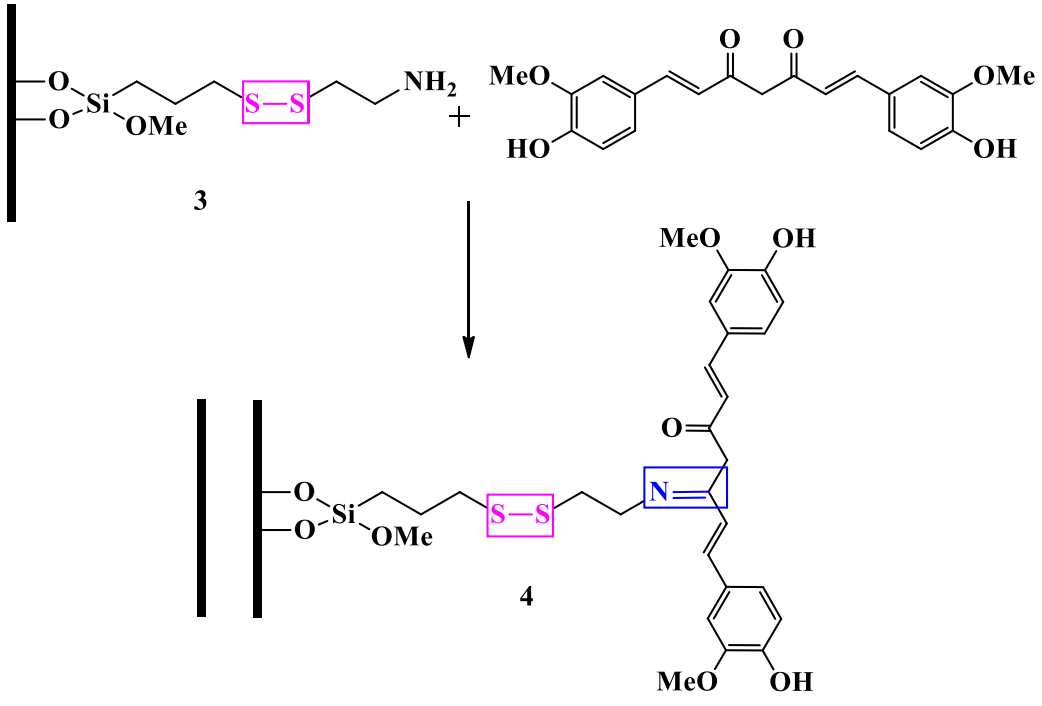

Scheme 1. Schematic representation of the synthesis of compound $\mathbf{3}$ and HNT-Cur (compound 4).

Table 1. Experimental conditions for the synthesis of compound 3.

\begin{tabular}{cccccccc}
\hline Entry & Compound & Heating & Solvent & $\begin{array}{c}\text { Time } \\
(\mathbf{h})\end{array}$ & $\begin{array}{c}\text { Tan } \\
(\boldsymbol{\delta})\end{array}$ & $\begin{array}{c}\text { Temperature } \\
\left({ }^{\circ} \mathbf{C}\right)\end{array}$ & $\begin{array}{c}\text { Loading } \\
(\boldsymbol{\%})^{\mathbf{a}}\end{array}$ \\
\hline 1 & $\mathbf{3}$ & Trad. & Methanol & 12 & 0.659 & 25 & 3.70 \\
2 & $\mathbf{3}$ & $\mathrm{MW}$ & Methanol & 1 & 0.659 & 50 & 3.57 \\
3 & $\mathbf{3}$ & $\mathrm{MW}$ & $/$ & 1 & $/$ & 50 & 3.97 \\
4 & $\mathbf{4}$ & Trad. & EtOH & 24 & 0.941 & 78 & 0.88 \\
5 & $\mathbf{4}$ & $\mathrm{MW}$ & $/$ & 1 & $/$ & 78 & 0.80 \\
6 & $\mathbf{4}$ & $\mathrm{MW}$ & $\mathrm{H} 2 \mathrm{O}$ & 1 & 0.123 & 78 & $/$ \\
7 & $\mathbf{4}$ & $\mathrm{MW}$ & $\mathrm{H}_{2} \mathrm{O} / \mathrm{EtOH}$ & 1 & & 78 & 0.40 \\
8 & $\mathbf{4}$ & $\mathrm{MW}$ & $\mathrm{EtOH}$ & 1 & 0.941 & 78 & 2.9 \\
9 & $\mathbf{4}$ & $\mathrm{MW}$ & $\mathrm{THF}$ & 1 & 0.047 & 78 & $/$ \\
\hline
\end{tabular}

afrom TGA results 


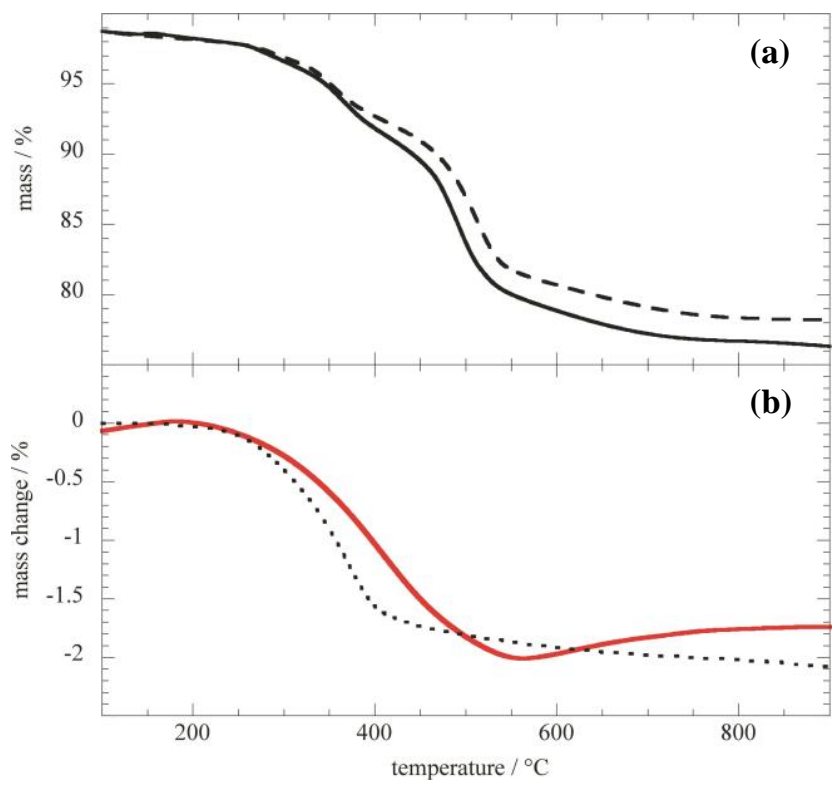

Figure 1. TG curves of a) compounds 3 (dashed line) and $\mathbf{4}$ (continue line); b) dependence on temperature of experimental mass loss difference between compounds $\mathbf{3}$ and $\mathbf{4}$ (solid line) and that calculated taking into account the Cur loading (dashed line).

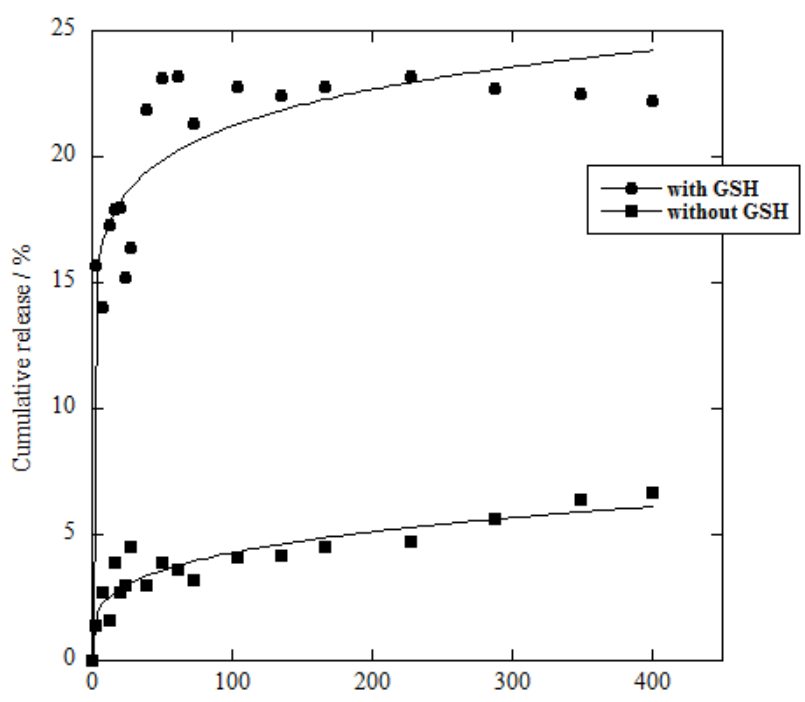

(a)

Time (min) 


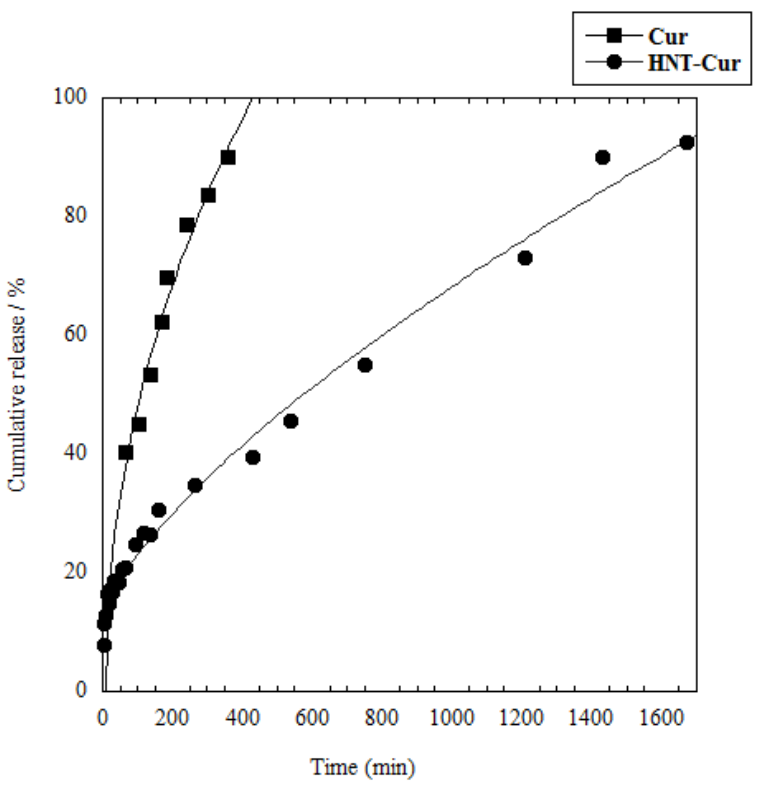

Figure 2. Kinetic release of curcumin from a) HNT-Cur prodrug at $37^{\circ} \mathrm{C}$ in a) $\mathrm{pH} 7.4$ phosphate buffer and b) $0.1 \mathrm{M}$ $\mathrm{HCl}$ solution (for comparison data about the kinetic release of pure curcumin from ref 37 ).

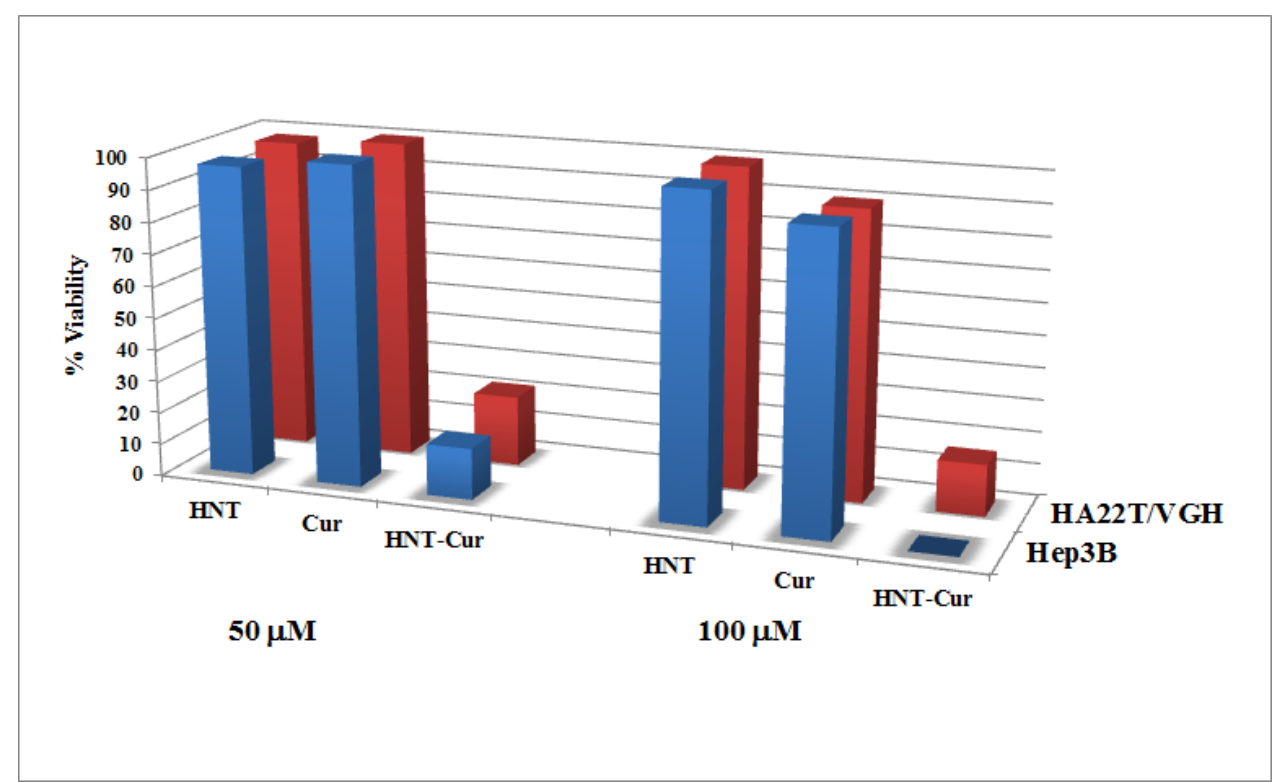

Figure 3. Graphical representation of cell viability of HA22T/VGH and Hep3B in presence of curcumin, HNT and HNT-Cur prodrug. 

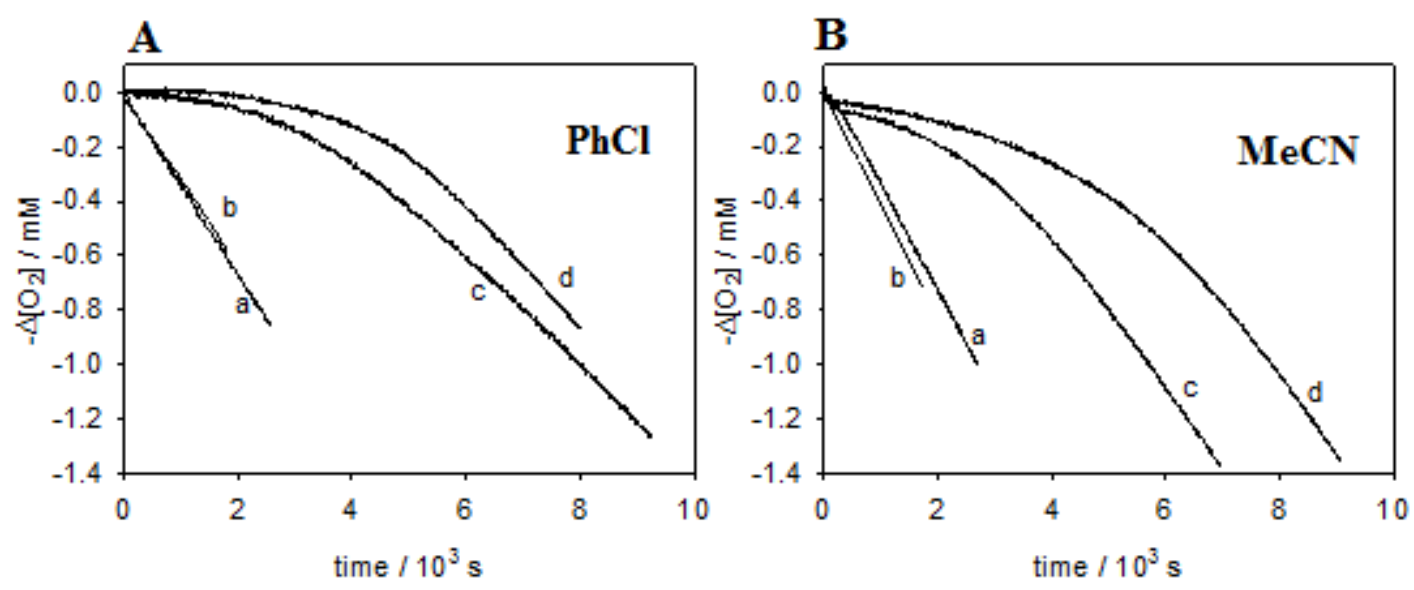

C

\begin{tabular}{ccccc}
\hline & PhCl & \multicolumn{2}{c}{ MeCN } \\
& $\boldsymbol{k}_{\text {inh }} \boldsymbol{M}^{-1} \boldsymbol{s}^{-\boldsymbol{1}}$ & $\boldsymbol{n}$ & $\boldsymbol{k}_{\text {inh }} \boldsymbol{M}^{-1} \boldsymbol{s}^{-\boldsymbol{t}}$ & $\boldsymbol{N}$ \\
\hline HNT & No inhibition & $/$ & No inhibition & $/$ \\
HNT -Cur & $(1.7 \pm 0.1) \times 10^{4}$ & $2.2 \pm 0.4$ & $(4.9 \pm 0.5) \times 10^{3}$ & $2.3 \pm 0.3$ \\
Curcumin & $(1.8 \pm 0.2) \times 10^{4}$ & $3.6 \pm 0.2$ & $(7.8 \pm 0.8) \times 10^{3}$ & $3.7 \pm 0.4$
\end{tabular}

Figure 4. Oxygen consumption traces measured during the autoxidation of cumene at $30{ }^{\circ} \mathrm{C}$ initiated by AIBN. Panel A: a) without antioxidant, b) HNT $0.08 \mathrm{mg} / \mathrm{mL}$, c) HNT-Cur: $0.08 \mathrm{mg} / \mathrm{mL}\left(2.9 \times 10^{-6} \mathrm{M}\right.$ in Cur), d) Cur $2.9 \times 10^{-6} \mathrm{M}$; solvent chlorobenzene. Panel B: a) without antioxidant, b) HNT $0.10 \mathrm{mg} / \mathrm{mL}$, c) HNT-Cur: 0.10 $\mathrm{mg} / \mathrm{mL}\left(2.9 \times 10^{-6} \mathrm{M}\right.$ in Cur), d) Cur $4.5 \times 10^{-6} \mathrm{M}$; solvent acetonitrile. Panel C: Antioxidant activity of HNT-Cur and of curcumin from autoxidation experiments.

Table 3. Number of radical trapped by HNT-Cur and of curcumin from oDPPH ${ }^{a}{ }^{a}$ assay, in acetonitrile at $298 \mathrm{~K}$.

\begin{tabular}{cc}
\hline Antioxidants & $\boldsymbol{n}^{\mathbf{b}}$ \\
\hline HNT & No inhibition \\
\hline HNT-Cur & \\
$13 / 1^{\text {c }}$ & $2 \pm 0.10$ \\
$10 / 1^{c}$ & $1.95 \pm 0.15$ \\
$6.5 / 1^{\mathrm{c}}$ & $1.75 \pm 0.15$ \\
\hline Curcumin & $4.0 \pm 0.5$
\end{tabular}

a 2,2-di(4-tert-octylphenyl)-1-picrylhydrazyl radical

bnumber of moles of DPPH• reduced per mole of curcumin (free or linked).

${ }^{c}$ number of moles of DPPH $\bullet /$ number of moles of antioxidant 